\title{
Higgs Transverse-Momentum Resummation in Direct Space
}

\author{
Pier Francesco Monni, ${ }^{1}$ Emanuele Re, ${ }^{2}$ and Paolo Torrielli ${ }^{3}$ \\ ${ }^{1}$ Rudolf Peierls Centre for Theoretical Physics, University of Oxford, Keble Road, Oxford OX1 3NP, United Kingdom \\ ${ }^{2}$ LAPTh, Université de Savoie, CNRS, BP 110, Annecy-le-Vieux F-74941, France \\ ${ }^{3}$ Dipartimento di Fisica, Università di Torino and INFN, Sezione di Torino, Via P. Giuria 1, I-10125 Turin, Italy
}

(Received 22 April 2016; published 17 June 2016)

\begin{abstract}
We propose a new approach to the resummation of the transverse-momentum distribution of a high-mass color-singlet system in hadronic collisions. The resummation is performed in momentum space and is free of kinematic singularities at small transverse momentum. We derive a formula accurate at the next-to-nextto-leading-logarithmic level, and present the first matched predictions to next-to-next-to-leading order for Higgs-boson production in gluon fusion at the LHC. This method can be adapted to all observables featuring kinematic cancellations in the infrared region.
\end{abstract}

DOI: 10.1103/PhysRevLett.116.242001

The determination of the properties of the scalar resonance discovered in 2012 by ATLAS and CMS [1,2] is central to the entire LHC physics program. At run II, owing to the increased collision energy and luminosity, the Higgsboson production rate will increase significantly. As a consequence, not only will the analyses already performed benefit from the increase in statistics, but soon it will become possible to study kinematic distributions in detail. Obtaining as accurate predictions as possible for the Higgs differential spectra is crucial, especially in view of the fact that the signal significance is very commonly optimized by categorizing candidate events according to their kinematic properties; therefore, only by means of precise predictions for the Higgs distributions can the increased statistics be fully exploited to extract physics results.

Among the Higgs production channels, the gluon-fusion mode is the dominant one at the LHC; among the most relevant kinematic distributions, the Higgs transverse momentum $p_{t}^{H}$ will be increasingly important in analyzing the forthcoming experimental results.

In gluon fusion the Higgs transverse momentum $p_{t}^{H}$ is defined as the inclusive vectorial sum over the transverse momenta of the recoiling QCD partons radiated off the incoming gluons. The fixed-order perturbative description of its differential distribution features large logarithms in the form $\alpha_{s}^{n} \ln ^{m}\left(m_{H} / p_{t}^{H}\right) / p_{t}^{H}$, with $m \leq 2 n-1$, which spoil the convergence of the series at small $p_{t}^{H}$. In order to obtain meaningful predictions in that phase-space region, such terms must be resummed to all orders in $\alpha_{s}$, so that the perturbative series can be recast in terms of dominant allorder towers of logarithms. The logarithmic accuracy is commonly defined at the level of the logarithm of the cumulative cross section, where one refers to the dominant terms $\alpha_{s}^{n} \ln ^{n+1}\left(m_{H} / p_{t}^{H}\right)$ as leading logarithms (LL), to terms $\alpha_{s}^{n} \ln ^{n}\left(m_{H} / p_{t}^{H}\right)$ as next-to-leading logarithms (NLL), to $\alpha_{s}^{n} \ln ^{n-1}\left(m_{H} / p_{t}^{H}\right)$ as next-to-next-to-leading logarithms (NNLL), and so on.
Such logarithms of the ratio $m_{H} / p_{t}^{H}$ have been resummed up to NNLL order in Refs. [3,4] using the formalism developed in Refs. [5,6], and in Ref. [7] using an effective-theory approach. A careful study of the theoretical uncertainties has also been carried out in Ref. [8], and a formalism to perform a joint $p_{t}^{H} /$ small- $x$ resummation has been presented in Ref. [9]. The differential distribution in fixed-order perturbation theory has been known for several years at next-to-leading order (NLO) [10-12], and has been recently improved through the computation of the Higgs-plus-one-jet cross section at next-to-next-to-leading order (NNLO) [13-16]. The inclusive cross section was computed at NNLO in Refs. [17-19] and recently at nextto-next-to-next-to-leading order $\left(\mathrm{N}^{3} \mathrm{LO}\right)$ in Refs. [20,21]. These results can be matched to a NNLL resummation in order to obtain a prediction which is accurate over the whole $p_{t}^{H}$ spectrum, analogously to what has been done for the leading-jet transverse momentum in Ref. [22].

All of the aforementioned resummations rely on an impact-parameter-space formulation, which is motivated by the fact that the observable naturally factorizes in this space as a product of the contributions from each individual emission. Conversely, in $p_{t}^{H}$ space one is unable to find, at a given order beyond LL, a closed analytic expression for the resummed distribution which is simultaneously free of any logarithmically subleading corrections and of singularities at finite $p_{t}^{H}$ values [23]. This fact has a simple physical origin: the region of small $p_{t}^{H}$ receives contributions both from configurations in which each of the transverse momenta of the radiated partons is equally small (Sudakov limit) and from configurations where $p_{t}^{H}$ tends to zero owing to cancellations among nonzero transverse momenta of the emissions. The latter mechanism becomes the dominant one at small $p_{t}^{H}$ and, as a result, the cumulative cross section in that region vanishes as $\mathcal{O}\left(p_{t}^{H 2}\right)$ rather than being exponentially suppressed [24]. If these effects are neglected in a resummation performed in 
transverse-momentum space, the latter would feature a geometric singularity at some finite value of $p_{t}^{H}$.

In this Letter we propose a new method that solves the problem in transverse-momentum space and extends the framework of Refs. [25,26] to treat all observables affected by the aforementioned kinematic cancellations. We obtain a NNLL-accurate formula for $p_{t}^{H}$ and match the result to the NNLO differential distribution for the first time.

The starting point for a NLL resummation is to consider an ensemble of partons $k_{1}, \ldots, k_{n}$, independently emitted off the initial-state gluons $\ell=1,2$. Momenta $k_{i}$ are parametrized as $k_{i}=z_{i}^{(1)} p_{1}+z_{i}^{(2)} p_{2}+\kappa_{t, i}$, where $p_{1,2}$ are the momenta of the incoming gluons and $\kappa_{t, i}$ is a spacelike four-vector, orthogonal to $p_{1}$ and $p_{2}$, i.e., $\kappa_{t, i}=\left(0, \vec{k}_{t, i}\right)$, such that $\kappa_{t, i}^{2}=-k_{t, i}^{2}$. By singling out the largest- $k_{t}$ emission (labeled by $k_{t, 1}$ ), the cumulative cross section can be written as

$$
\begin{aligned}
\Sigma\left(p_{t}^{H}\right) & =\int_{0}^{p_{t}^{H}} d p_{t}^{\prime} \frac{d \sigma\left(p_{t}^{\prime}\right)}{d p_{t}^{\prime}}=\sigma_{0} \int_{0}^{\infty}\left\langle d k_{1}\right\rangle R^{\prime}\left(k_{t, 1}\right) e^{-R\left(\epsilon k_{t, 1}\right)} \\
& \times \sum_{n=0}^{\infty} \frac{1}{n !} \prod_{i=2}^{n+1} \int_{\epsilon k_{t, 1}}^{k_{t, 1}}\left\langle d k_{i}\right\rangle R^{\prime}\left(k_{t, i}\right) \Theta\left(p_{t}^{H}-\left|\vec{q}_{n+1}\right|\right)
\end{aligned}
$$

where $\sigma_{0}$ denotes the Born cross section, $\vec{q}_{n+1}=\sum_{j=1}^{n+1} \vec{k}_{t, j}$, and the measure $\left\langle d k_{i}\right\rangle$ is defined in Eq. (2). The radiator $R\left(\epsilon k_{t}\right)$ reads [27]

$$
R\left(\epsilon k_{t}\right)=4 \int_{\epsilon k_{t}}^{m_{H}} \frac{d k_{t}^{\prime}}{k_{t}^{\prime}}\left(\frac{\alpha_{s}^{\mathrm{CMW}}\left(k_{t}^{\prime}\right)}{\pi} C_{A} \ln \frac{m_{H}}{k_{t}^{\prime}}-\alpha_{s}\left(k_{t}^{\prime}\right) \beta_{0}\right),
$$

where $\alpha_{s}^{\mathrm{CMW}}\left(k_{t}\right)$ in the double-logarithmic part indicates that the strong coupling is expressed in the CataniMarchesini-Webber (CMW) scheme [28], which ensures the correct treatment of nonplanar soft corrections at NLL accuracy in processes with two hard emitters. The independent-emission amplitude squared and its phase space are parametrized in Eq. (1) as [25,26]

$$
[d k] M^{2}(k)=\frac{d k_{t}}{k_{t}} \frac{d \phi}{2 \pi} R^{\prime}\left(k_{t}\right) \equiv\langle d k\rangle R^{\prime}\left(k_{t}\right),
$$

where $R^{\prime}\left(k_{t}\right)=-k_{t} d R\left(k_{t}\right) / d k_{t}$. The bounds in the $\left\langle d k_{i}\right\rangle$ integrals of Eq. (1) apply to the $k_{t, i}$ variables, while all azimuthal integrals are evaluated in the range $[-\pi, \pi]$. In Eq. (1) the parton luminosity implicit in $\sigma_{0}$ is evaluated at a fixed factorization scale $\mu_{F}$, while a complete NLL description requires a scale of the order of $k_{t, 1}$. Since this approximation is irrelevant for the present discussion, we ignore it for the moment, and account for the proper treatment of the luminosity only in the main result [Eq. (9)] of this Letter.

The NLL transverse-momentum resummation then proceeds by expanding the various $k_{t, i}$ 's of Eq. (1) around the observable $p_{t}^{H}$, retaining terms only up to NLL in the cumulative cross section. This amounts to writing

$$
\begin{aligned}
R\left(\epsilon k_{t, 1}\right) & =R\left(p_{t}^{H}\right)+R^{\prime}\left(p_{t}^{H}\right) \ln \frac{p_{t}^{H}}{\epsilon k_{t, 1}}+\cdots, \\
R^{\prime}\left(k_{t, i}\right) & =R^{\prime}\left(p_{t}^{H}\right)+\cdots,
\end{aligned}
$$

where neglected terms contribute at most to NNLL order in $p_{t}^{H}$ space. The second term in the expansion of $R\left(\epsilon k_{t, 1}\right)$ plays the role of virtual contribution, canceling the infrared divergences associated with the real emissions to all orders in $\alpha_{s}$. With these replacements, Eq. (1) becomes

$$
\begin{aligned}
\Sigma\left(p_{t}^{H}\right)= & \sigma_{0} e^{-R\left(p_{t}^{H}\right)} \int_{0}^{\infty}\left\langle d k_{1}\right\rangle R^{\prime}\left(p_{t}^{H}\right)\left(\frac{p_{t}^{H}}{k_{t, 1}}\right)^{-R^{\prime}\left(p_{t}^{H}\right)} \\
& \times \epsilon^{R^{\prime}\left(p_{t}^{H}\right)} \sum_{n=0}^{\infty} \frac{1}{n !} \prod_{i=2}^{n+1} \int_{\epsilon k_{t, 1}}^{k_{t, 1}}\left\langle d k_{i}\right\rangle R^{\prime}\left(p_{t}^{H}\right) \Theta\left(p_{t}^{H}-\left|\vec{q}_{n+1}\right|\right),
\end{aligned}
$$

which evaluates to

$$
\Sigma\left(p_{t}^{H}\right)=\sigma_{0} e^{-R\left(p_{t}^{H}\right)} e^{-\gamma_{E} R^{\prime}\left(p_{t}^{H}\right)} \frac{\Gamma\left(1-R^{\prime}\left(p_{t}^{H}\right) / 2\right)}{\Gamma\left(1+R^{\prime}\left(p_{t}^{H}\right) / 2\right)} .
$$

Equation (5) reproduces the result of Ref. [23]; the geometric singularity at $R^{\prime}\left(p_{t}^{H}\right)=2$ invalidates the result near the peak of the distribution, as a consequence of the dominance of the aforementioned cancellation mechanism over the usual Sudakov suppression. This comes about since in the asymptotic limit $p_{t}^{H} \ll k_{t, 1}$, the second line of Eq. (4) scales as $\left(p_{t}^{H} / k_{t, 1}\right)^{2}$ [29], which causes the cumulative cross section to diverge at $R^{\prime}\left(p_{t}^{H}\right)=2$.

The issue hides behind expansion Eq. (3), which was performed with the aim of neglecting subleading effects: such an expansion is indeed valid only in the region where $p_{t}^{H} / k_{t, 1} \gtrsim 1$, while it cannot be applied when $p_{t}^{H} / k_{t, 1} \rightarrow 0$. A natural solution can be achieved by using an impactparameter-space formulation $[24,30]$, since the conjugate variable $b$ correctly describes the vectorial nature of the $p_{t}^{H} \rightarrow 0$ limit.

However, the problem can also be overcome in direct space by simply expanding $k_{t, i}$ around $k_{t, 1}$ instead of $p_{t}^{H}$; namely,

$$
\begin{aligned}
R\left(\epsilon k_{t, 1}\right) & =R\left(k_{t, 1}\right)+R^{\prime}\left(k_{t, 1}\right) \ln \frac{1}{\epsilon}+\cdots, \\
R^{\prime}\left(k_{t, i}\right) & =R^{\prime}\left(k_{t, 1}\right)+\cdots
\end{aligned}
$$

The resulting cumulative cross section reads

$$
\begin{aligned}
\Sigma\left(p_{t}^{H}\right)= & \sigma_{0} \int_{0}^{\infty}\left\langle d k_{1}\right\rangle R^{\prime}\left(k_{t, 1}\right) e^{-R\left(k_{t, 1}\right)} \epsilon^{R^{\prime}\left(k_{t, 1}\right)} \\
& \times \sum_{n=0}^{\infty} \frac{1}{n !} \prod_{i=2}^{n+1} \int_{\epsilon k_{t, 1}}^{k_{t, 1}}\left\langle d k_{i}\right\rangle R^{\prime}\left(k_{t, 1}\right) \Theta\left(p_{t}^{H}-\left|\vec{q}_{n+1}\right|\right) .
\end{aligned}
$$

Since by construction $k_{t, i} / k_{t, 1} \leq 1$, the expansion in Eq. (6) is always justified, and the exponential factor regularizes the $p_{t}^{H} / k_{t, 1} \rightarrow 0$ limit. Equation (7) can be effectively 
interpreted as a resummation of the large logarithms $\ln \left(m_{H} / k_{t, 1}\right)$, and the logarithmic order is defined in terms of the latter. This formulation provides a correct description of both mechanisms that drive the limit $p_{t}^{H} \rightarrow 0$, and it can be shown [31] that Eq. (7) reproduces the correct powersuppressed scaling in this region [24]. The corresponding formal accuracy in terms of the logarithms $\ln \left(m_{H} / p_{t}^{H}\right)$ will be the same, and the result differs from Eq. (5) by subleading logarithmic terms.

The above treatment can be systematically extended to NNLL. Since the observable considered here is fully inclusive over QCD radiation, the initial equation (1) already contains most of the NNLL contributions, as shown in Ref. [26]. More specifically, one should modify Eq. (7) introducing the NNLL radiator $R_{\mathrm{NNLL}}$, which is the same as for the jet-veto resummation [32], and retaining the next term in the expansion [Eq. (6)], which involves the second derivative of the radiator, $R^{\prime \prime}\left(k_{t, 1}\right) \equiv-k_{t, 1} d R^{\prime}\left(k_{t, 1}\right) / d k_{t, 1}$; the parton luminosity is to be evaluated at a scale of the order of $k_{t, 1}$, as will be detailed in Ref. [31]. It is, furthermore, convenient to neglect $\mathrm{N}^{3} \mathrm{LL}$ terms in the $R^{\prime}\left(k_{t, 1}\right)$ and $R^{\prime \prime}\left(k_{t, 1}\right)$ functions. We introduce the notation

$$
\begin{aligned}
R^{\prime}\left(k_{t, 1}\right) & =\hat{R}^{\prime}\left(k_{t, 1}\right)+\delta \hat{R}^{\prime}\left(k_{t, 1}\right)+\cdots, \\
R^{\prime \prime}\left(k_{t, 1}\right) & =\hat{R}^{\prime \prime}\left(k_{t, 1}\right)+\cdots,
\end{aligned}
$$

where the functions $\hat{R}^{\prime}\left(k_{t, 1}\right)$ and $\delta \hat{R}^{\prime}\left(k_{t, 1}\right)$ are NLL and NNLL, respectively, the neglected terms are at most of order $\alpha_{s}^{n} \ln ^{n-2}\left(m_{H} / k_{t, 1}\right)$, and $\hat{R}^{\prime \prime}\left(k_{t, 1}\right)$ indicates the derivative of $\hat{R}^{\prime}\left(k_{t, 1}\right)$. The expressions for all these functions can be found in the Appendix of Ref. [32]. The NNLL cumulative cross section is thus conveniently recast as

$$
\begin{aligned}
\Sigma\left(p_{t}^{H}\right) & =\int_{0}^{\infty}\left\langle d k_{1}\right\rangle\left[\epsilon^{\hat{R}^{\prime}\left(k_{t, 1}\right)} \sum_{n=0}^{\infty} \frac{1}{n !} \prod_{i=2}^{n+1} \int_{\epsilon k_{t, 1}}^{k_{t, 1}}\left\langle d k_{i}\right\rangle \hat{R}^{\prime}\left(k_{t, 1}\right)\right]\left\{\partial_{L}\left[-e^{-R_{\mathrm{NNLL}}\left(k_{t, 1}\right)} \mathcal{L}\right] \Theta\left(p_{t}^{H}-\left|\vec{q}_{n+1}\right|\right)\right. \\
& \left.+e^{-R\left(k_{t, 1}\right)} \hat{R}^{\prime}\left(k_{t, 1}\right) \int_{\epsilon k_{t, 1}}^{k_{t, 1}}\left\langle d k_{s}\right\rangle\left[\left(\delta \hat{R}^{\prime}\left(k_{t, 1}\right)+\hat{R}^{\prime \prime}\left(k_{t, 1}\right) \ln \frac{k_{t, 1}}{k_{t, s}}\right) \hat{\mathcal{L}}-\partial_{L} \hat{\mathcal{L}}\right]\left[\Theta\left(p_{t}^{H}-\left|\vec{q}_{n+1, s}\right|\right)-\Theta\left(p_{t}^{H}-\left|\vec{q}_{n+1}\right|\right)\right]\right\},
\end{aligned}
$$

with $\vec{q}_{n+1, s}=\vec{q}_{n+1}+\vec{k}_{t, s}$. The above formula can be evaluated by means of Monte Carlo methods. The parton luminosity $\mathcal{L}$ is defined as

$$
\begin{aligned}
\mathcal{L}= & \frac{\alpha_{s}^{2}\left(\mu_{R}\right)}{576 \pi v^{2}} \tau \sum_{i, j} \int_{\tau}^{1} \frac{d x_{1}}{x_{1}} \int_{x_{1}}^{1} \frac{d z_{1}}{z_{1}} \int_{\tau / x_{1}}^{1} \frac{d z_{2}}{z_{2}}[H C C]_{g g ; i j} \\
& \times f_{i}\left(x_{1} / z_{1}, e^{-L} \mu_{F}\right) f_{j}\left(\tau / x_{1} / z_{2}, e^{-L} \mu_{F}\right),
\end{aligned}
$$

where $\mu_{R}$ is the renormalization scale, $\tau=m_{H}^{2} / s, v$ is the vacuum expectation value of the Higgs field, and $L=\ln \left(Q / k_{t, 1}\right)$, the resummation scale $Q$ being introduced as shown in Refs. [4,32]. The factor $[H C C]$ is defined as

$$
\begin{aligned}
{[H C C]_{g g ; i j} } & =H_{g}^{H}\left[\alpha_{s}\left(\mu_{R}\right), \mu_{R}, Q, m_{H}\right] \\
& \times\left[C_{g i}\left(z_{1} ; \alpha_{L}, \mu_{R}, \mu_{F}, Q\right) C_{g j}\left(z_{2} ; \alpha_{L}, \mu_{R}, \mu_{F}, Q\right)\right. \\
& \left.+G_{g i}\left(z_{1} ; \alpha_{L}, \mu_{R}, \mu_{F}\right) G_{g j}\left(z_{2} ; \alpha_{L}, \mu_{R}, \mu_{F}\right)\right], \quad(10)
\end{aligned}
$$

where $\alpha_{L}=\alpha_{s}\left(\mu_{R}\right) /\left[1-2 \alpha_{s}\left(\mu_{R}\right) \beta_{0} L\right]$. The product in Eq. (10) is further expanded neglecting constant terms beyond $\mathcal{O}\left(\alpha_{s}^{2}\right)$. The functions $H_{g}^{H}, C_{i j}$, and $G_{i j}$ are deduced using the results of Ref. [33], after including the proper scale dependences. The NLL luminosity $\hat{\mathcal{L}}$ is obtained from $\mathcal{L}$ by setting $[H C C]_{g g ; i j}=\delta_{g i} \delta_{g j} \delta\left(1-z_{1}\right) \delta\left(1-z_{2}\right)$, and it reproduces the Born cross section $\sigma_{0}$ in the limit $L \rightarrow 0$.

The various contributions to Eq. (9) are described in what follows. The first line includes all NNLL corrections to the hardest emission $k_{1}$; this reflects the arguments that led to Eq. (7), properly extended to NNLL. The corrections to the remaining emissions are encoded in the second line of Eq. (9), where only a single emission $k_{s}$ of the ensemble is corrected. This is implemented in Eq. (9) by computing the difference between the observable evaluated using all emissions, including the modified one $k_{s}$, and the observable obtained by neglecting $k_{s}$. Since these configurations give at most a NNLL correction, it suffices to use the NLL luminosity $\hat{\mathcal{L}}$ and radiator $R\left(k_{t, 1}\right)$ in the second line of Eq. (9). Finally, the term proportional to $\partial_{L} \hat{\mathcal{L}}$ accounts for the luminosity contribution to the NNLL hard-collinear correction, described by a Altarelli-Parisi-evolution step between $\epsilon k_{t, 1}$ and $k_{t, 1}$. The corresponding contribution for the first emission is encoded in the first line of Eq. (9), where terms beyond NNLL are included in order to reproduce the exact differential for the hardest-emission probability. The latter are physical contributions; namely, they are a subset of the subleading terms that would be generated by retaining higher orders in the resummation.

As a check of Eq. (9), we have expanded it around $k_{t, 1}=p_{t}^{H}$, neglecting $\mathrm{N}^{3} \mathrm{LL}$ terms in $\ln \left(m_{H} / p_{t}^{H}\right)$. This approximation is the source of the singularity in Eq. (5), but it contains all the correct NNLL terms at a given fixed order in $\alpha_{s}$, which can be used as a powerful test of the accuracy of our result. Equation (9) reproduces the analytic result reported in the Appendix of Ref. [32] at NNLL.

As a phenomenological application of Eq. (9), we perform a matching to the $\mathrm{N}^{3} \mathrm{LO}$ cumulant, which is obtained by combining the total $\mathrm{N}^{3} \mathrm{LO}$ cross section [21] and the NNLO Higgs-plus-jet cross section [13]. We perform an additive matching, and unitarity at high $p_{t}^{H}$ is restored by introducing the modified logarithms

$$
\ln \left(Q / k_{t, 1}\right) \rightarrow 1 / p \ln \left[\left(Q / k_{t, 1}\right)^{p}+1\right],
$$


where we choose $p=2$. (This choice is made only for consistency with the literature we compare with. A study on the optimal choice of $p$ is left for future work.) We consider $13 \mathrm{TeV}$ LHC collisions, with $m_{H}=125 \mathrm{GeV}$, and PDF 4LHC15 [34] parton densities at NNLO. The central prediction uses $\mu_{R}=\mu_{F}=m_{H}$, and $Q=m_{H} / 2$. The perturbative uncertainty for all predictions is estimated by varying both $\mu_{R}$ and $\mu_{F}$ by a factor of 2 in either direction while keeping $1 / 2 \leq \mu_{R} / \mu_{F} \leq 2$. Moreover, for central $\mu_{R}$ and $\mu_{F}$ scales, we vary the resummation scale $Q$ by a factor of 2 in either direction. Our results are obtained in the heavy-top effective theory; the inclusion of quarkmass effects, which poses no problems in our framework, is, however, beyond the scope of the present discussion.

To validate our result, in the main panel of Fig. 1 we show the comparison of our prediction for the Higgs-transversemomentum spectrum at NNLL + NLO to that obtained with HqT $[4,35]$. As expected, we observe a very good agreement over the entire $p_{t}^{H}$ range between these two results, which have the same perturbative accuracy. Our NNLL + NLO prediction is moderately higher in the peak of the distribution, and lower at intermediate $p_{t}^{H}$ values, although this pattern may slightly change with different central-scale choices. These small differences have to do with the different treatment of subleading effects in the two resummation methods. The agreement of the two results, both for the central scale and for the uncertainty band, is even more evident in the lower inset of Fig. 1, which displays the ratio of the various distributions, each normalized to its central-scale inclusive rate, to our normalized central NNLL + NLO curve.

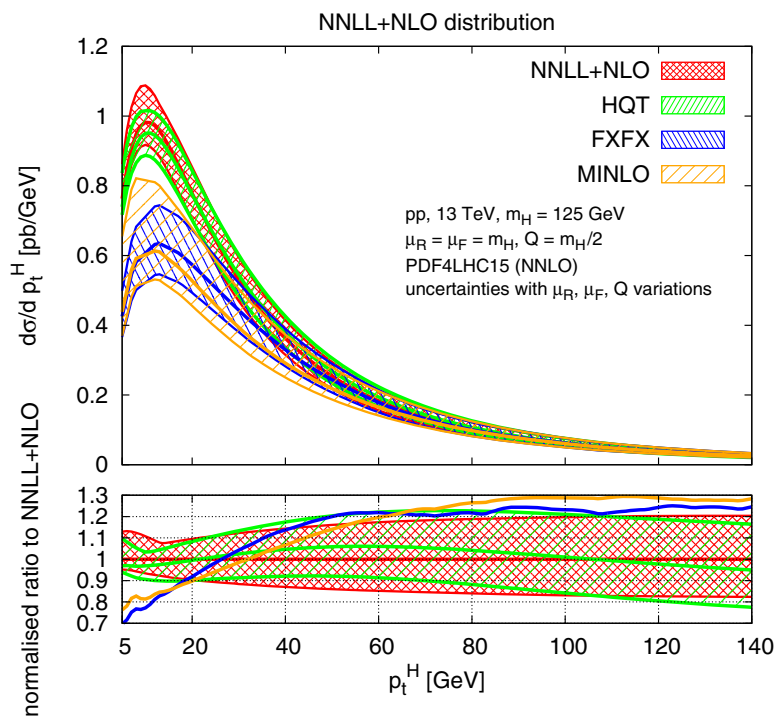

FIG. 1. Comparison of the Higgs $p_{t}^{H}$ NNLL + NLO prediction as obtained in this Letter (red) to HQT (green). For reference, the predictions obtained with MINLO at NLO (orange) and FXFX (blue) are shown. Lower panel: Ratio of the various distributions, normalized to their respective central-scale inclusive cross sections, to the central NNLL + NLO prediction. Uncertainty bands are shown only for the resummed results.
For comparison, Fig. 1 also reports the $p_{t}^{H}$ distribution obtained with the NLO version of POWHEG+MiNLO [36-38], and with the MADGRAPH5_AMC@NLO+FXFX [39-41] event generators, using default renormalization and factorization scales for the two methods (in FXFX a merging scale $\mu_{Q}=$ $m_{H} / 2$ has been employed). Both generators are interfaced to Pythia8.2 [42], without including hadronization, underlying event, and primordial $k_{\perp}$ (whose impact has been checked to be fully negligible for this observable), and use PDF 4LHC15 parton densities at NLO. By inspecting the normalized ratios shown in the lower panel, one observes that the shape of the Monte Carlo predictions deviates significantly from the NNLL + NLO results at $p_{t}^{H} \lesssim 60 \mathrm{GeV}$.

Figure 2 shows the comparison of the matched NNLL + NNLO result to the NNLL + NLO and the fixed-order NNLO predictions. The NNLO prediction shown there and used for the matching is obtained by running the code of Ref. [15] with a cut of $p_{t}^{H}>10 \mathrm{GeV}$, and no phase-space cuts on jets.

The inclusion of the NNLO corrections leads to a $10 \%-$ $15 \%$ increase in the matched spectrum for $p_{t}^{H}>15 \mathrm{GeV}$, and to a reduction in the uncertainty at medium-high $p_{t}^{H}$, from $15 \%-20 \%$ to $10 \%$. For $p_{t}^{H} \gtrsim 40 \mathrm{GeV}$, the NNLOmatched distribution and its uncertainty reduce to the NNLO prediction, while the impact of resummation on the fixed order becomes increasingly important for $p_{t}^{H} \lesssim 40 \mathrm{GeV}$, reaching about $25 \%$ at $p_{t}^{H}=15 \mathrm{GeV}$. At low $p_{t}^{H}$ the uncertainty of the two matched results is identical, since this region is dominated by resummation.

In this Letter we have presented a new method, entirely formulated in momentum space, for the resummation of the transverse momentum of a color-singlet final state in hadronic collisions. We have used it to obtain the first NNLL + NNLO prediction for the Higgs-boson transversemomentum spectrum at the LHC. Higher-order logarithmic

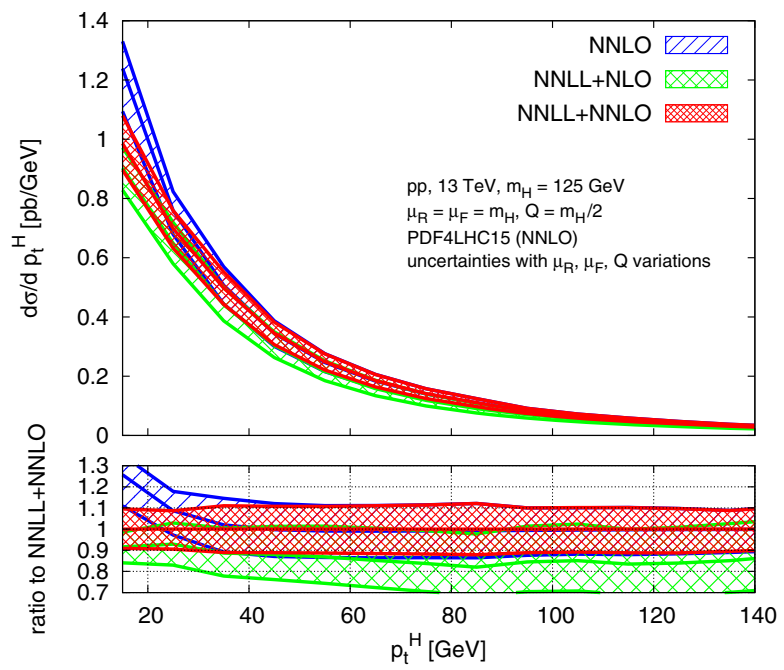

FIG. 2. Higgs $p_{t}^{H}$ at NNLL + NNLO (red), NNLL + NLO (green), and NNLO (blue). Lower panel: Ratio of the three predictions to the NNLL + NNLO. 
corrections beyond NNLL can be systematically included within this framework. Our approach does not rely on any specific factorization theorem, and, therefore, it can be generalized to treat any observable featuring kinematic cancellations in the infrared region-like, for instance, $\phi^{*}$ in Drell-Yan pair production [43] or the oblateness in electron-positron annihilation-as well as to compute any other observable which can be treated with the methods of Refs. [25,26]. Notably, this paves the way for formulating a simultaneous resummation for the Higgs and the leadingjet transverse momenta at NNLL.

We are very grateful to $\mathrm{F}$. Caola for providing us with the NNLO distributions used in this work and for checking the fixed-order results, and to G. Salam for very useful discussions on some aspects of the topic treated here, as well as for help with the use of the HOPPET code [44]. We also thank F. Dulat for providing us with the $\mathrm{N}^{3} \mathrm{LO}$ total cross sections used in the results, and A. Banfi, L. Magnea, and G. Zanderighi for carefully reading the manuscript and for providing valuable comments. We acknowledge the hospitality of CERN's Theory Department while part of this work was carried out. P. F. M. was partially supported by the Swiss National Science Foundation (SNF) under Grant No. PBZHP2-147297. P. F. M. and E. R. have benefitted from the ERC Grant No. 614577 HICCUP. The work of P.T. has received funding from the European Union Seventh Framework programme for research and innovation under the Marie Curie Grant Agreement No. 6094022020 researchers: Train to Move (T2M).

[1] G. Aad et al. (ATLAS Collaboration), Phys. Lett. B 716, 1 (2012).

[2] S. Chatrchyan et al. (CMS Collaboration), Phys. Lett. B 716, 30 (2012).

[3] G. Bozzi, S. Catani, D. de Florian, and M. Grazzini, Phys. Lett. B 564, 65 (2003).

[4] G. Bozzi, S. Catani, D. de Florian, and M. Grazzini, Nucl. Phys. B737, 73 (2006).

[5] J. C. Collins, D. E. Soper, and G. F. Sterman, Nucl. Phys. B250, 199 (1985).

[6] S. Catani, D. de Florian, and M. Grazzini, Nucl. Phys. B596, 299 (2001).

[7] T. Becher and M. Neubert, Eur. Phys. J. C 71, 1665 (2011).

[8] D. Neill, I. Z. Rothstein, and V. Vaidya, J. High Energy Phys. 12 (2015) 097.

[9] S. Marzani, Phys. Rev. D 93, 054047 (2016).

[10] D. de Florian, M. Grazzini, and Z. Kunszt, Phys. Rev. Lett. 82, 5209 (1999).

[11] V. Ravindran, J. Smith, and W. L. Van Neerven, Nucl. Phys. B634, 247 (2002).

[12] C. J. Glosser and C. R. Schmidt, J. High Energy Phys. 12 (2002) 016.

[13] R. Boughezal, F. Caola, K. Melnikov, F. Petriello, and M. Schulze, Phys. Rev. Lett. 115, 082003 (2015).

[14] R. Boughezal, C. Focke, W. Giele, X. Liu, and F. Petriello, Phys. Lett. B 748, 5 (2015).
[15] F. Caola, K. Melnikov, and M. Schulze, Phys. Rev. D 92 , 074032 (2015).

[16] X. Chen, T. Gehrmann, N. Glover, and M. Jaquier, arXiv:1604.04085.

[17] R. V. Harlander and W. B. Kilgore, Phys. Rev. Lett. 88, 201801 (2002).

[18] C. Anastasiou and K. Melnikov, Nucl. Phys. B646, 220 (2002).

[19] V. Ravindran, J. Smith, and W. L. van Neerven, Nucl. Phys. B665, 325 (2003).

[20] C. Anastasiou, C. Duhr, F. Dulat, F. Herzog, and B. Mistlberger, Phys. Rev. Lett. 114, 212001 (2015).

[21] C. Anastasiou, C. Duhr, F. Dulat, E. Furlan, T. Gehrmann, F. Herzog, A. Lazopoulos, and B. Mistlberger, J. High Energy Phys. 05 (2016) 058.

[22] A. Banfi, F. Caola, F. A. Dreyer, P. F. Monni, G. P. Salam, G. Zanderighi, and F. Dulat, J. High Energy Phys. 04 (2016) 049.

[23] S. Frixione, P. Nason, and G. Ridolfi, Nucl. Phys. B542, 311 (1999).

[24] G. Parisi and R. Petronzio, Nucl. Phys. B154, 427 (1979).

[25] A. Banfi, G. P. Salam, and G. Zanderighi, J. High Energy Phys. 03 (2005) 073.

[26] A. Banfi, H. McAslan, P. F. Monni, and G. Zanderighi, J. High Energy Phys. 05 (2015) 102.

[27] A. Banfi, G. P. Salam, and G. Zanderighi, J. High Energy Phys. 06 (2012) 159.

[28] S. Catani, B. R. Webber, and G. Marchesini, Nucl. Phys. B349, 635 (1991).

[29] A. Banfi, G. P. Salam, and G. Zanderighi, J. High Energy Phys. 01 (2002) 018.

[30] Y. L. Dokshitzer, D. Diakonov, and S. I. Troian, Phys. Lett. B 79, 269 (1978).

[31] P. F. Monni, E. Re, and P. Torrielli (to be published).

[32] A. Banfi, P. F. Monni, G. P. Salam, and G. Zanderighi, Phys. Rev. Lett. 109, 202001 (2012).

[33] S. Catani and M. Grazzini, Eur. Phys. J. C 72, 2013 (2012); 72, 2132(E) (2012).

[34] J. Butterworth et al., J. Phys. G 43, 023001 (2016).

[35] D. de Florian, G. Ferrera, M. Grazzini, and D. Tommasini, J. High Energy Phys. 11 (2011) 064.

[36] P. Nason, J. High Energy Phys. 11 (2004) 040.

[37] S. Alioli, P. Nason, C. Oleari, and E. Re, J. High Energy Phys. 06 (2010) 043.

[38] K. Hamilton, P. Nason, C. Oleari, and G. Zanderighi, J. High Energy Phys. 05 (2013) 082.

[39] S. Frixione and B. R. Webber, J. High Energy Phys. 06 (2002) 029.

[40] J. Alwall, R. Frederix, S. Frixione, V. Hirschi, F. Maltoni, O. Mattelaer, H.-S. Shao, T. Stelzer, P. Torrielli, and M. Zaro, J. High Energy Phys. 07 (2014) 079.

[41] R. Frederix and S. Frixione, J. High Energy Phys. 12 (2012) 061.

[42] T. Sjöstrand, S. Ask, J. R. Christiansen, R. Corke, N. Desai, P. Ilten, S. Mrenna, S. Prestel, C. O. Rasmussen, and P.Z. Skands, Comput. Phys. Commun. 191, 159 (2015).

[43] A. Banfi, S. Redford, M. Vesterinen, P. Waller, and T. R. Wyatt, Eur. Phys. J. C 71, 1600 (2011).

[44] G. P. Salam and J. Rojo, Comput. Phys. Commun. 180, 120 (2009). 